\title{
Secondary Injection of Gases into a Supersonic Flow
}

\author{
Edward E. Zukoski* and Frank W. Spaid $\dagger$ \\ California Institute of Technology, Pasadena, Calif.
}

\begin{abstract}
The flow field around the injection port for secondary injection of a gas normal to a supersonic stream has been studied in a series of wind-tunnel experiments. The experiments were conducted at freestream Mach numbers of 1.38 to 4.54. Gaseous nitrogen, argon, and helium were used as injectants. New information concerning pressure fields, concentration fields, and shock shapes was obtained. A scale parameter has been calculated, based on a simple, inviscid model of the flow field. This scale parameter gives a good general correlation of the data. Use of this scale parameter allows prediction of a simple scaling law for the side forces generated by secondary injection. This side-force scaling law is in approximate agreement with existing rocket motor test results.
\end{abstract}

\section{Nomenclature}

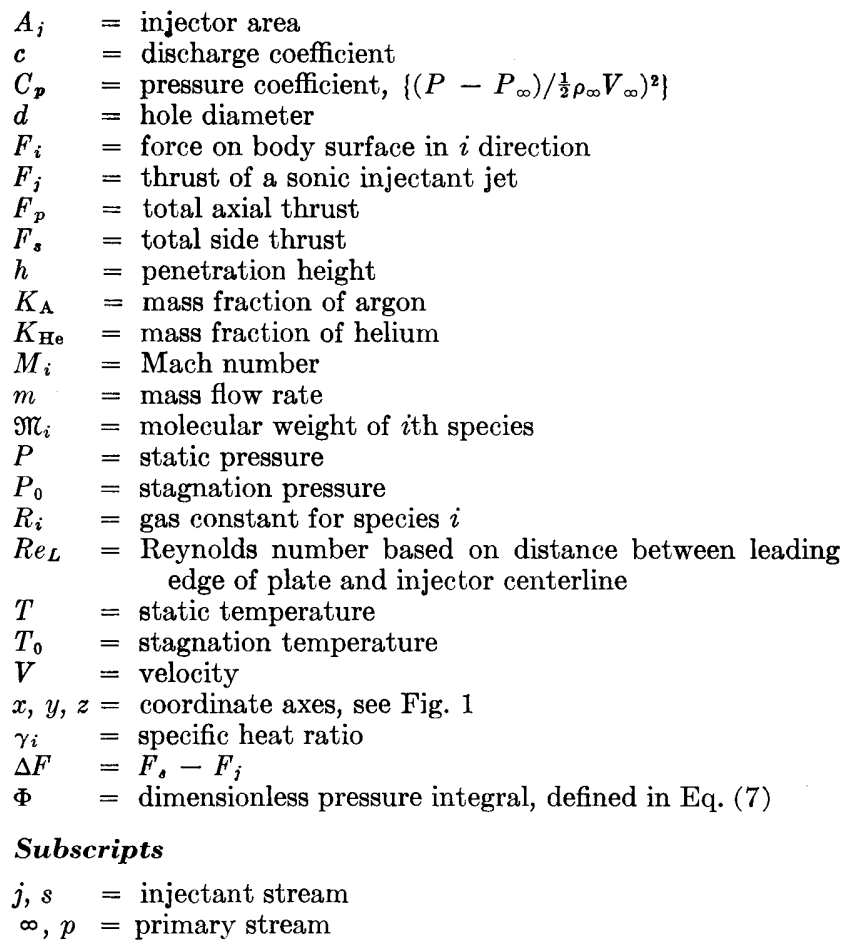

\section{Introduction}

$\mathbf{T}$ HE description of the flow field set up by the injection of a secondary gas into a supersonic primary flow is a problem of current engineering interest. Flows of this type occur during thrust vector control of rocket motors, during jet reaction attitude control of vehicles moving through the atmosphere, and during fuel injection into a supersonic burner. In all these applications, when a gas is injected into the primary flow, the injected material acts as an obstruction to the primary flow and, as such, produces a strong

Presented as Preprint 64-110 at the AIAA Solid Propellant Rocket Conference, Palo Alto, Calif., January 29-31, 1964; revision received July 10, 1964. This research work was supported by the Daniel and Florence Guggenhiem Jet Propulsion Center of the California Institute of Technology and by NASA Contract No. NAS 7-100, through the Solid Rockets Section of the Jet Propulsion Laboratory, California Institute of Technology.

* Associate Professor of Jet Propulsion, Daniel and Florence Guggenheim Jet Propulsion Center. Associate Fellow Member AIAA.

$\dagger$ Graduate Student, Mechanical Engineering, Daniel and Florence Guggenheim Jet Propulsion Center. Member AIAA. shock wave in the primary flow. This shock interacts with the boundary layer on the wall to form a complex flow system that produces both high- and low-pressure regions in the neighborhood of the injector.

Although much work has been done to investigate the phenomena associated with injection (e.g., Ref. 2) it is still not well understood. The goal of the present study has been to obtain fundamental information concerning the processes of interaction which occur during secondary injection and, in particular, to determine similarity rules for the important phenomena. The situation chosen for experimental and theoretical study is the sonic injection of a gas through a wall and normal to a primary flow that is uniform and rectilinear outside a wall boundary layer. In the present paper, a model for the interaction region is presented with the purpose of developing a scaling parameter, and the results are compared with experimental data obtained by the authors and by other workers.

\section{Description of Experiments}

A series of experiments were conducted in the 2.5-in. supersonic wind tunnel at the California Institute of Technology (CIT), in which gaseous nitrogen, argon, and helium were injected through orifices in the test-section side walls at test-section Mach numbers of 2.56 and 1.38. Tests were conducted with both laminar and turbulent boundary layers in the region of the injector at the first Mach number, and with a turbulent boundary layer at the second. Experimental data consisted of test-section flow conditions, schlieren photographs, static pressure distributions on the test section wall in the injection region, concentration measurements in the flow, and injectant total pressure and mass flow rate.

In addition to this work, experiments were conducted in the 20 -in. supersonic wind tunnel at the Jet Propulsion Laboratory (JPL). These experiments were part of a series conducted by J. F. Newton Jr. and M. W. Dowdy ${ }^{1}$ and the authors. Gaseous nitrogen was injected through a sonic orifice $0.100 \mathrm{in}$. in diameter normal to the surface of a sharpedged flat plate, 7.00 in. to the rear of the leading edge. Tests were conducted at test-section Mach numbers of 4.54, $3.50,2.61$, and 2.01 and at several freestream stagnation pressures. At Mach numbers 4.54 and 3.50, the boundary layer on the plate near the injection port was always laminar, and at Mach number 2.01, it was always turbulent. The experimental data consisted of schlieren and shadowgraph pictures, tunnel and injection conditions, and static pressure measurements on the plate in the injection region.

\section{Description of the Flow Field}

Before discussing the analytic model or experimental work, it is convenient to review briefly the general features of the 


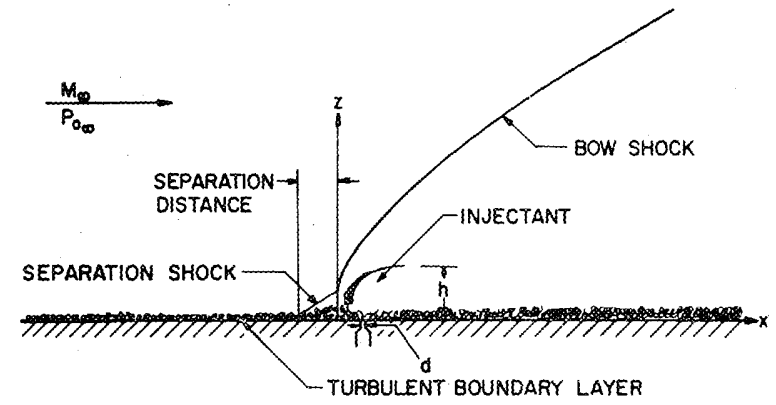

Fig. 1a Sketch of the flow field, umbulent boundary layer.

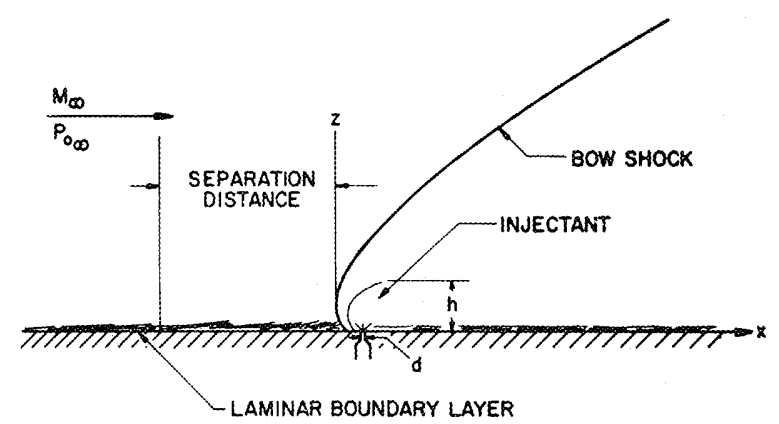

Fig. Ib Sketch of the flow field, laminar boundary layer.

flow near the injector. A crude picture of the flow field produced by injection through an orifice can be obtained by examination of schlieren and shadowgraph pictures of the interaction region. Sketches taken from shadowgraph photographs and schlieren photographs of typical flow conditions are shown in Figs. 1 and 2 . The sketches are seale drawings of two shadowgraph pictures taken of flow over the JPL flat-plate model. The schlieren photographs show the effects of injection through the CIT tunnel wall. The scale drawings and photographs were taken at different freestream stagnation pressures but at approximately the same ratio of jet stagnation pressure to freestream stagnation pressure, and the freestream Mach number was 2.61 .

For these examples, the injected material enters through a circular orifice, with a static pressure much higher than that in the undisturbed primary flow. The flow is sonic at the injector and expands rapidly through a strong Prandt Meyer fan. The interaction of the two streams produces a strong bow wave on the upstream side of the injector, and the shock-induced pressure field turns the injectant until it moves approximately parallel to the wall.

The bow shock-wave/boundary-layer interaction produces a region of boundary-layer separation upstream of the shock. For the case of a turbulent boundary layer (see Figs. 1a and 2a), the separated region is short, and the oblique shock produced by separation is usually sufficiently strong to be observed. When the boundary layer is laminar (Figs. 1b and $2 \mathrm{~b}$ ), the separated region is much larger, and the angle between the separated flow and the wall is never more than a few degrees.

Additional information can be obtained from static wallpressure measurements. As would be expected, the wall pressure rises above the ambient value near the intersection of the shock system and the wall. The pressure rise dies out as the distance downstream from the injector increases. Immediately downstream of the injector is a region in which both injectant and primary flows separate from the wall and in which the pressure is far below the ambient value.

Some details of the flow near the injector are also shown on the photographs. One feature that usually is seen for nitrogen and argon injection, but not for helium injection, and that is apparently the region of maximum concentration of the injectant, has the appearance of a streamline of the injectant. This feature is shown in Fig. 1 and can also be seen in the schlieren photographs of Fig. 2. Determination of the maximum distance between this feature and the wall (see Fig. 1) gives a simple visual measure of the penetration of the secondary fluid into a primary flow. Therefore, this distance is called the penetration height. Figures 1 and 2 illustrate flow conditions in which the distance of this feature from the wall is much larger than a characteristic boundary-layer dimension. Because of the difference in the shadowgraph and schlieren photographs, it was found convenient to use different flow features in measuring the penetration heights. Hence, the data obtained by use of the two techniques are not directly comparable.

\section{Analytic Model}

In investigating the shock patterns produced by secondary injection, one is reminded of shock shapes produced by blunt axisymmetric bodies. This fact suggested that some insight into the scaling laws for secondary injection could be obtained by setting up a simple model for a solid body which. would give a shock pattern similar to that produced by injeetion.

The scheme used here is to pick a shape for the nose of the equivalent body and then to calculate the characteristic dimension of the nose by balancing the drag of the nose section against the momentum flux of the injectant. The analysis is carried out for the momentum flux and force acting parallel to the wall, and the control volume chosen for the analysis is the nose section of the equivalent body which lies close to the injectant orifice. It is expected that such a simple model may give a useful description of a scaling parameter without necessarily including an accurate description of all the details of the flow. The salient features of the flow utilized in the model are the bluff nature of the effective interference body, the separated region immediately downstream of the injector, and the fact that the mixing between the injectant and other gas may be small in the immediate neighborhood of the injector.

The coordinate system used in describing the model is illustrated in Fig. 1. The $x-z$ plane is shown with the $y$ axis perpendicular to the page. The origin of the coordinate system has been chosen so that the $y=0$ plane includes the center of the injector and so that the origin lies at the intersection of the bow shock wave with the wall.

For the purposes of this model it is assumed 1) that a sonic jet is injected into a uniform supersonic flow with no wall boundary layer, 2) that no mixing occurs between the injectant and either the primary flow or the separated flow near the injector, 3) that the interface between the injectant and primary flows is a quarter sphere followed by an axisymmetric half body, and 4) that the interface between the separated flow downstream of the injector and the injectant always lies inside the surface described in item 3.

The force balance is made on the control volume formed by the quarter-spherical nose, which is located with one plane surface in the $x-y$ plane and the other in the $y-z$ plane. It is assumed 5) that the pressure forces on the sphere due to primary flow can be calculated by use of modified Newtonian flow, 6) that the injectant expands isentropically to the ambient pressure with its velocity parallel to the wall at the downstream face of the sphere, and 7) that the contribution to the momentum flux across the $y-z$ plane due to flow in the separated region downstream of the injector can be neglected.

A brief description of the derivation of the equation for the penetration height is given here (a more detailed treatment occurs in Ref. 16). First, consider the flow over the spherical nose. For Newtonian flow calculations, with the modificam tions suggested by Lees, 3 " the pressure coefficient on the surface of the body is given by $C_{p} / C_{p}^{*}=\sin ^{2} \alpha / \sin ^{2} \alpha^{*}$, where $\alpha$ is the angle between the local tangent to the surface and 
the undisturbed flow direction and must lie between 0 and $\pi / 2$. Here, $C_{p}^{*}$ and $\alpha^{*}$ are evaluated at the nose of the body. Hence, $\alpha^{*}=\pi / 2$, and $C_{p}{ }^{*}$ is the pressure coefficient corresponding to the stagnation pressure behind a normal shock and is a function of the freestream Mach number and specific heat ratio. The total axial force on the spherical surface, obtained by integrating the pressure force in the $x$ direction over the spherically shaped nose of the equivalent body, is given by

$$
F_{x}=(\pi / 8) M_{\infty}^{2} \gamma_{\infty} C_{p}^{*} h^{2} P_{\infty}
$$

The force on the inside surface of the nose is found by making a momentum balance of the injectant flowing through the control volume. Because the jet is injected normal to the wall, the momentum change is equal to the total $x$ momentum of the injected gas passing through the downstream face of the control volume. At this point the injectant (see assumption 6) has expanded isentropically to the loeal ambient pressure. Thus, the velocity at this point, $V_{2}$, is given by the expression for isentropic expansion from a stagnation pressure $P_{0 j}$ to a static pressure $P_{\infty}$.

The mass flux of the injectant $m_{i}$ is equal to the mass flux at the injector port, which is calculated by assuming sonic flow through a circular orifice of equivalent diameter $\left\{d(c)^{1 / 2}\right\}$. The momentum flux is just $m_{j} V_{j}$, and the force balance is therefore given by

$$
F_{x}=m_{j} V_{j}
$$

All the parameters in this equation are known except the radius of the sphere, and therefore Eq. (2) can be solved for this parameter with the result

$$
\begin{aligned}
& \frac{h}{d(c)^{1 / 2}}=\left(\left(\frac{1}{M_{\infty}}\right)\left(\frac{P_{0 j}}{P_{\infty}} \frac{\gamma_{j}}{\gamma_{\infty}} \frac{2}{C_{p}^{*}}\right)^{1 / 2} \times\right. \\
& \left.\left\{\frac{2}{\gamma_{i}-1}\left(\frac{2}{\gamma_{j}+1}\right)^{\left(\gamma_{j}+1\right) /\left(\gamma_{j}-1\right)}\left[1-\left(\frac{P_{\infty}}{P_{0 i}}\right)^{\left(\gamma_{j}-1\right) / \gamma_{j}}\right]\right\}^{1 / 4}\right)
\end{aligned}
$$

It is proposed that the radius $h$ can be used as a measure of the seale of the disturbance produced by injection. Note that, although the expression given in Eq. (3) contains no adjustable constants, the exact correspondence between. values calculated from $\mathrm{Eq}$. (3) and any measured feature of the flow, such as the penetration height, is purely fortuitous. However, it is to be expected that changes in seale of flow features will be proportional to changes in $h$.

The variations of the terms in the brackets of Eq. (3) and that of $C_{p}{ }^{*}$ with $M_{\infty}$ or $\gamma_{\infty}$ are not very rapid. Hence, the most important variation of the scale parameter is approximately given by

$$
h \propto\left[d(c)^{1 / 2}\right]\left(1 / M_{\infty}\right)\left(P_{0 i} / P_{\infty}\right)^{1 / 2}
$$

In terms of the mass flow rate of injectant $m_{i}$, Eq. (4) can be written as

$$
h \propto\left(1 / M_{\infty}\right)\left(m_{j} / P_{\infty}\right)^{1 / 2}\left(R_{i} T_{0 j}\right)^{1 / 4}
$$

Note that, for simplicity, the complex dependence on $\gamma_{j}$ and $\gamma_{\infty}$ has been omitted here. Equations (4) and (5) are useful as long as $\left(P_{\infty} / P_{0 i}\right) \ll 1$ and $M_{\infty}>2$.

It should be noted that the functional form of Eq. (3) is not sensitive to the shape assumed for the nose. For example, the derivation has been carried out for elliptical nose shapes with eccentricities ranging between 0 and 0.98 . The equations for $h$ obtained by this calculation had the same functional form given in Eq. (3) and differed from it by a multiplicative constant that depended on the eccentricity and that only changed by a factor of 2.3 for the range of eccentricities given previously.

Sinee the boundary layer has not been considered at all in this analysis, it would be expected that $h$ would have to be much larger than a characteristic boundary-layer dimension in the immediate vicinity of the jet, i.e., a characteristic thickness of the boundary layer in the region of maximum separation, in order for the analysis to be applicable.

However, there is a difference between the observed flow field and the model which seems to make this limitation somewhat less severe. As the injectant expands just after leaving the orifice, it is initially conical in shape, so that the actual obstruction shape as viewed from the front is probably similar to that of a frustrum of a cone, with its small end resting on the wall, which is capped by a sphere. This shape would be expected to produce considerably less of an obstacle to the boundary layer than a quarter sphere of the same projected area.

To provide a basis for comparison, some experiments with solid objects of the same shape as that postulated in the analytic model have been conducted. These objects were attached to the nozzle wall, and schlieren photographs were taken at a test-section Mach number of 2.56. Photographs with the same shock shape were compared. The separation produced with both laminar and turbulent wall boundary layers was always considerably more extensive than that produced by injection, thus verifying the previous supposition about the applicability of the model.

\section{Presentation of Experimental Data}

In the following sections, results are given of experiments concerning the flow field geometry, concentration measurements, and static pressure measurements on the wall. The data are presented in terms of space coordinates normalized by the scale parameter $h$, which is calculated from Eq. (3). This mode of presentation was used to facilitate the verification of the proposed scaling law.

\section{Penetration Height}

In most of the schlieren photographs taken with secondary injection of argon and nitrogen into air, a distinct feature

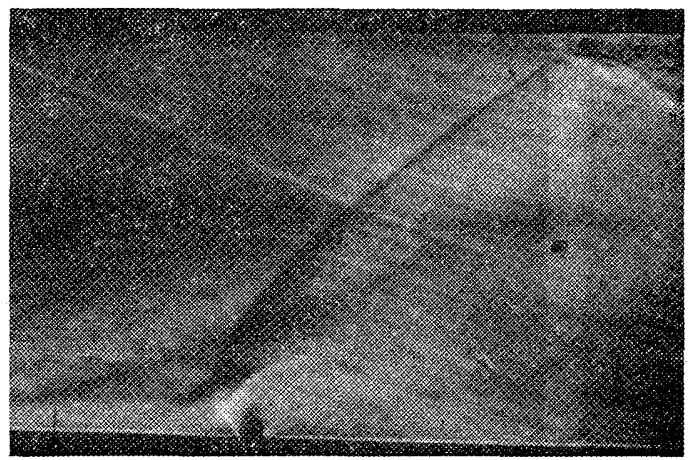

Fig. 2a Schlieren photograph, turbulent boundary layer.

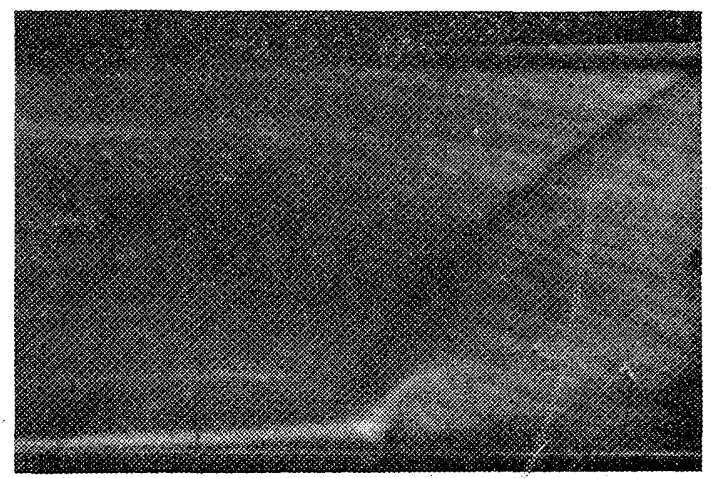

Fig. $2 b$ Schlieren photograph, laminar boundary layer. 


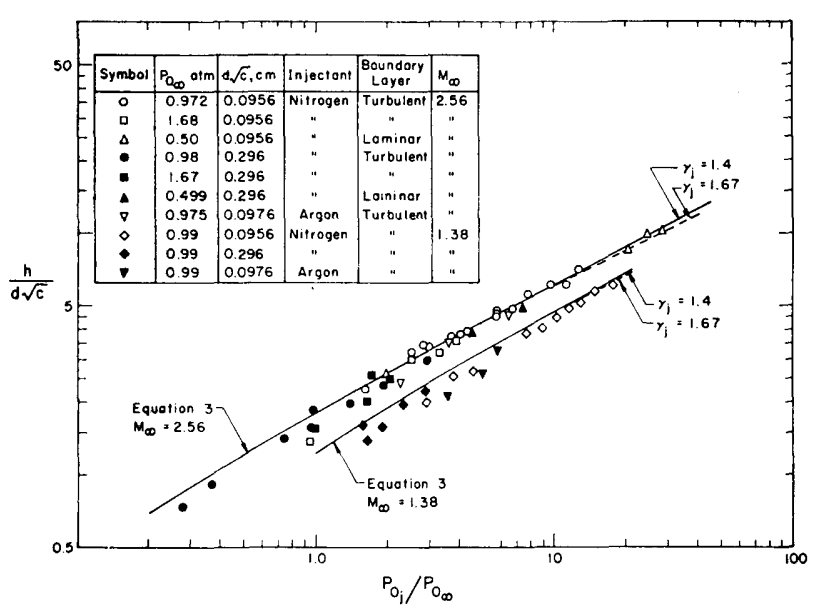

Fig. 3 Penetration of secondary jet into primary flow, nozzle-wall data.

appears which looks like the top or outer boundary of the jet. Although this feature, shown in Fig. 1, is probably the line of maximum concentration of injectant rather than the jet boundary, it has been selected as being characteristic of the scale of the disturbance produced by the jet and hence is called the penetration height of the jet. That flow fields for different injection rates should be geometrically similar is, of course, an assumption that must be verified.

Values of the penetration heights determined directly from schlieren photographs made in the CIT facility are shown in Fig. 3. Here, penetration heights normalized by the equivalent injector diameter are given as a function of the ratio of injectant to primary stream stagnation pressures; data are presented for two Mach numbers. Nitrogen and argon were used as injectants, and experiments were conducted with two injector diameters.

No dependence on injector molecular weight or specific heat ratio was noticed when penetration heights for a given total pressure ratio were compared. The data shown in Fig. 3 were obtained with laminar and turbulent boundary layers. It is particularly interesting to note that measured values of the penetration height were not noticeably dependent on the state of the boundary layer, which was about 0.2 in. thick for these tests.

Theoretical values of penetration heights are also shown in Fig. 3 for both Mach numbers. The agreement between experiment and theory is good over the whole pressure ratio range studied, and the dependence on specific heat ratio, molecular weight, and Mach number is correctly predicted.

Values of penetration height were also obtained in the JPL facility. The measurements were made from shadow-

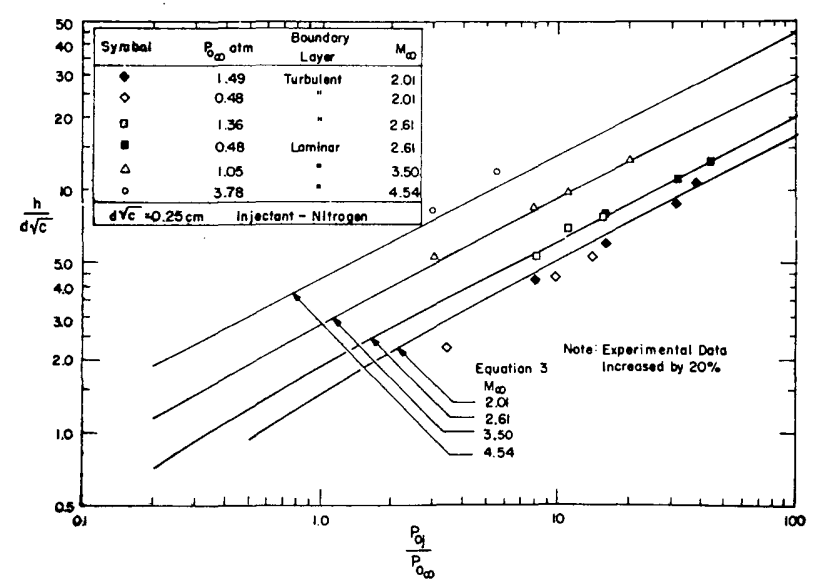

Fig. 4 Penetration of secondary jet into primary flow, flat-plate data. graph pictures, and it was convenient to use a different feature of the flow than was used in the schlieren photographs. Comparison of these data with those obtained in the CIT facility showed that the JPL flat-plate results were about $20 \%$ lower than the comparable CIT nozzle-wall data. In comparing the data with values predicted from the model for the scale parameter, it should be remembered that the features examined on either schlieren or shadowgraph pictures are not the jet boundaries themselves, and hence that either the calculated or the measured penetration heights can only be viewed as being proportional to the actual characteristic scale of the flow.

Comparison of the flat-plate data and the results of Eq. (3) are shown in Fig. 4. To facilitate comparison, the measured values are increased by $20 \%$. The dependence on Mach number and pressure ratio of the calculated and measured values are in good agreement, although the experimental data have a slightly more rapid variation with Mach number than that predicted from Eq. (3).

Although the good agreement between the predicted and measured values is fortuitous, the accurate prediction of the dependence of penetration height on injector diameter, injection pressure, and primary-flow Mach number indicates that the value of the scale parameter given by Eq. (3) is a useful measure of the scale of the interaction phenomena occurring near the injector. In addition, the agreement suggests that the model does include the pertinent physical phenomena and that the gross features of the flow near the injector can be characterized by a single dimension that is proportional to $h$.

\section{Shock Shapes}

As a further check on the suggestion that $h$ is a characteristic dimension of the flow field, the shapes of the bow shock waves, as seen from the side, were determined from schlieren and shadowgraph pictures from both the flat-plate and nozzlewall data and for argon, helium, and nitrogen injectants. In all cases, when the shock coordinates were normalized by values of $h$ calculated from Eq. (3), the normalized coordinates were found to form a family of curves, one curve for each value of $M_{\infty}$. For a given Mach number, the data agreed very well for the three injectants. Hence, it is evident that the characteristic dimension of interaction is substantially independent of molecular weight.

\section{Concentration Profiles}

A more critical check on the proposed scaling law is given by examination of the flow pattern of the injectant. The mixing of the injectant and primary flows has been examined by making analyses of gas samples drawn from various loca-

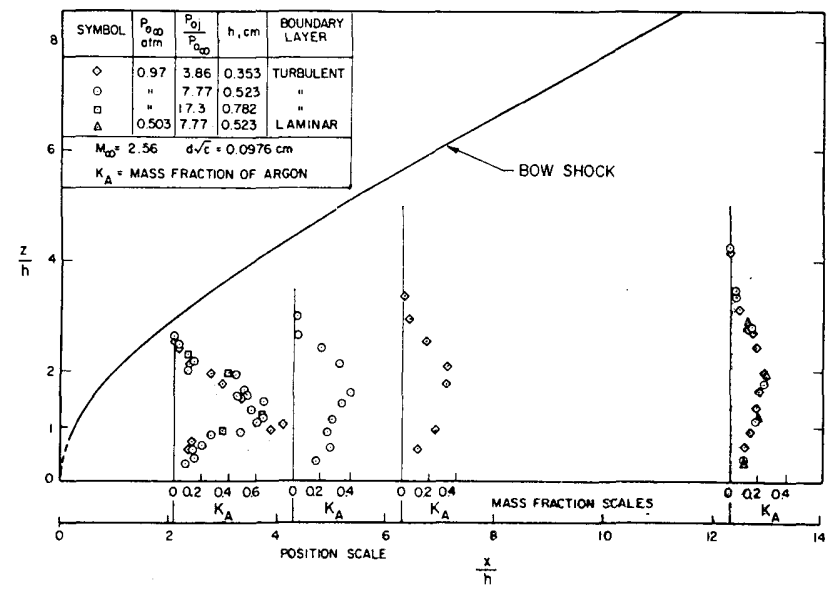

Fig. 5 Concentration measurements with argon injection in the plane $(y / h)=0$, downstream region. 
tions in the flow field. Measurements were taken at Mach number 2.56 in the nozzle-wall injection system with argon and helium injectants. The positions most thoroughly studied lie in the $x-z$ plane, $y \equiv 0$; a few positions for other values of $y$ were also examined.

Data obtained with argon injectant in the $y \equiv 0$ plane are shown in Fig. 5. Here, the origin of each concentration profile is superimposed at the appropriate position on a plot of the $x-z$ plane which also shows the bow shock wave. The probe that was used to obtain these concentration measurements was $0.079-\mathrm{cm}$. o.d.

The data of Fig. 5 show that, near the injector, the profile is sharply curved on the lower side of the maximum, but that downstream of $(x / h) \approx 4$ the profile is roughly Gaussian except for a slight wall interference effect. It is obvious that the observed and calculated penetration height corresponds much more closely to the line of maximum concentration than to the outer edge of the injectant stream.

For $(x / h) \approx 2$, the maximum concentration was found to be less than 0.80 ; thus, even this close to the injector, the injectant is already substantially mixed with the primary flow. Farther downstream mixing is slower. From this result, it is obvious that the no-mixing approximation made in the model can only be useful close to the injector. However, $h$ is a measure of the scale of the injectant flow, and hence it is reasonable to expect that it would be the characteristic dimension for the mixing process too.

Measurements made on planes other than $y=0$ are shown in Fig. 6 for two values of $(x / h)$. Here, lines of constant concentration in the $(y / h)-(z / h)$ plane are presented. The solid points shown on the figure were obtained by direct interpolation from concentration profiles, and the dotted lines are based on a double interpolation of the data given in Ref. 16. The shapes of the two plots are roughly similar. The kidney-shaped cross section seen in the concentration profiles of Fig. 6 suggests that a vortex is shed from either side of the injectant jet. The vortex filaments appear to be roughly parallel to the wall, and with vorticity such that, near the wall, primary gas is swept in toward the centerline of the flow, i.e., toward $(y / h)=0$. This type of vortex structure has been observed by other workers for the case of subsonic injection into a subsonic stream. ${ }^{5}$ Such vortices may explain the steep gradients in concentration observed at the $(x / h) \approx$ 2 position.

Similar data obtained with helium injectant are shown in Fig. 7. As would be expected, these curves, presented in terms of mass fractions, are not identical with the argon data, since the two situations are not directly comparable.

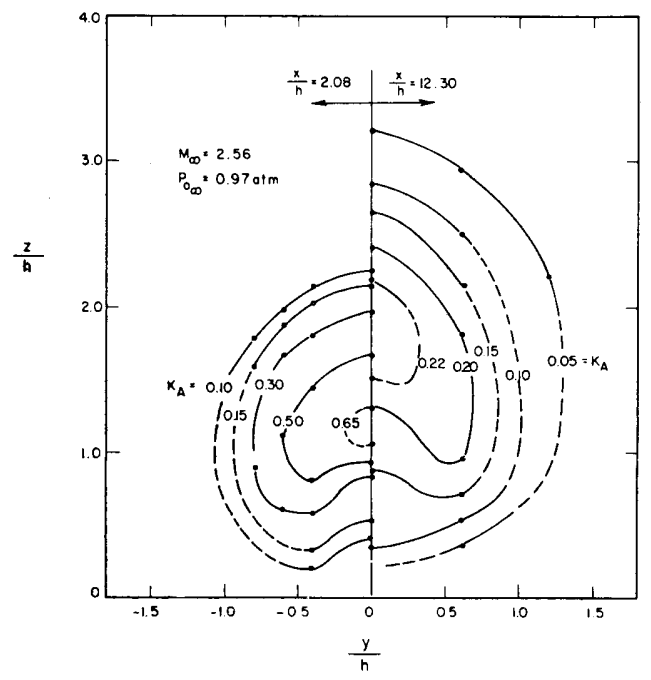

Fig. 6 Concentration contours in the planes $(x / h)=$ 2.08 and $(x / h)=12.30$

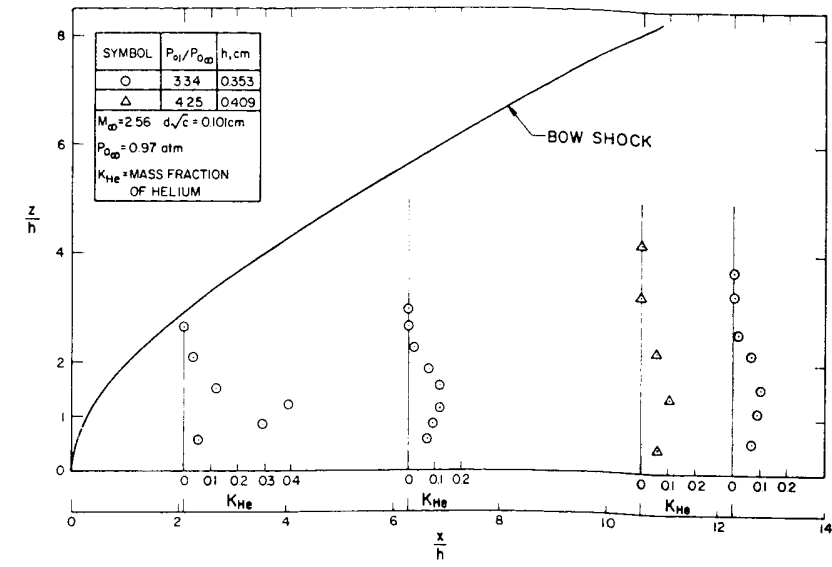

Fig. 7 Concentration measurements with helium injection in the plane $(y / h)=0$.

Although the general shapes of the curves shown in Figs. 5 and 7 are quite similar, such features as jet width and distance of maximum concentration line from the wall are definitely smaller for the helium case.

Argon data are presented in Figs. 5 and 6 for stagnation pressure ratios that give a $2.2: 1$ change in penetration height. The normalized concentration profiles shown in these figures are almost identical over this scale change. The data are also insensitive to the state of the boundary layer, since both laminar and turbulent layers are included. Hence, it is apparent that the scaling rule given by Eq. (3) for a particular gas is valid for the mixing process, too, when changes in scale by not more than a factor of 2 are considered; the good agreement of the data suggests that much larger scale changes could be adequately treated.

\section{Wall Pressure Distribution}

The experimental results discussed up to this point concern the gross structure of the flow field produced by secondary injection. It has been shown that these features of the flow are approximately independent of the state of the boundary layer and that a simple model of the flow leads to the calculation of a single characteristic dimension $h$, which is a satisfactory scaling parameter. In contrast, when examining the flow field near the wall, the state of the boundary layer is very important because of its influence on the interaction between the bow shock and the boundary layer (see Figs. 1 and 2 ).

Static pressure data obtained in the CIT and JPL facilities are shown in Figs. 8-13. Values of the pressure change produced by injection and normalized by the primary-flow static

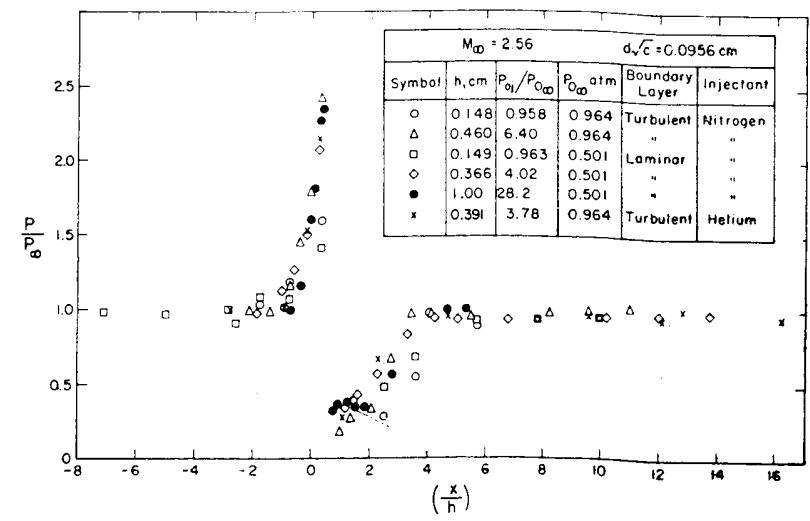

Fig. 8 Nozzle-wall static pressure measurements in the plane $(y / h)=0$ at $M_{\infty}=2.56$ with nitrogen and helium injectants. 

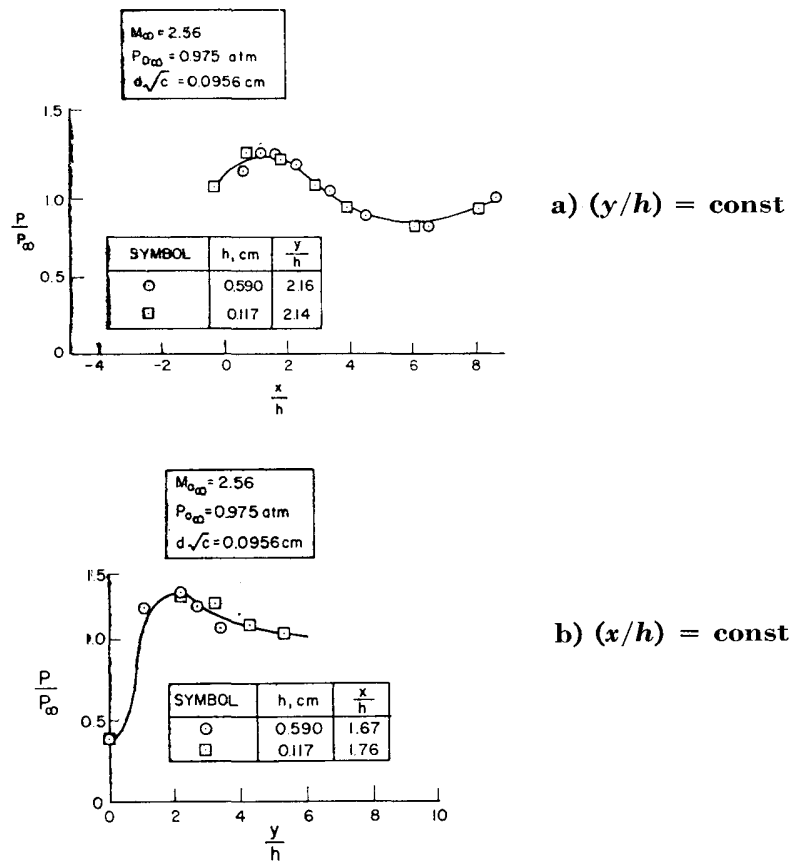

b) $(x / h)=$ const

Fig. 9 Nozzle-wall static pressure measurements in offaxis planes with nitrogen injection.

pressure are given as functions of the position coordinates normalized by the calculated penetration height. Consider first the results for $M=2.56$ shown in Figs. 8 and 9 . In Fig. 8, data are shown for pressures measured along the $x$ axis. In general, the pressure increases rapidly between $-1 \leq(x / h) \leq-\frac{1}{2}$ on the upstream side of the injector port, falls to less than half the ambient around the port, and rises to the ambient value in the region $3 \leq(x / h) \leq 4$. When the boundary layer is laminar, the region of increased pressure extends somewhat farther upstream of the shock because of separation.

Off-axis data are shown in Fig. 9 for conditions corresponding to the turbulent boundary-layer data of Fig. 8. The data are for cuts along both $y=$ const and $x=$ const lines and serve to give a rough picture of the off-axis pressure distribution. As would be expected, pressure extremes are found along the $x$ axis and die off with distance away from this axis.

A wide range of injector parameters are covered by the data presented in Figs. 8 and 9 . For example, the change in

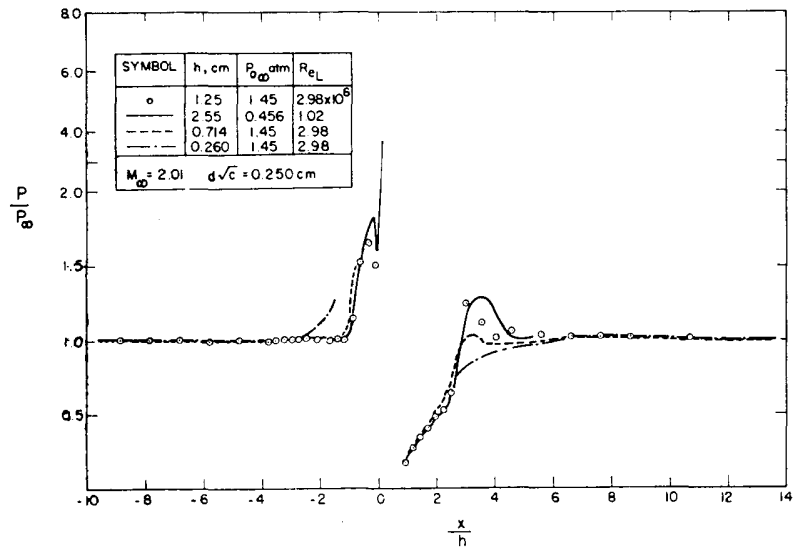

Fig. 10 Flat-plate static-pressure measurements in the plane $(y / h)=0$ at $M_{\infty}=2.01$ with nitrogen injection: turbulent boundary layer. total pressure ratios used here produces a $6.8: 1$ variation in the penetration height; data with both laminar and turbulent boundary layers are presented, and helium and nitrogen injectants are used. In view of this wide variation of parameters, the correlation of the data of Figs. 8 and 9 by use of the normalization factor $h$ is satisfactory.

The pressure distributions obtained at different Mach numbers for the flat-plate model are shown in Figs. 10-13. Again, it was not possible to present all of the data points; each figure includes one set of data points, and the scatter in the data for which only average curves are given is similar to that of the data that are presented.

At Mach 2.61, the pressure distributions obtained with a turbulent boundary layer are in good agreement with the nozzle-wall data of Fig. 8. A curve representing the average of the CIT data of Fig. 8 is given in Fig. 11 to facilitate this comparison. One of the differences between the nozzle-wall and the flat-plate data for the case of turbulent boundary layers was that separation could usually be observed in the flat-plate pressure-distribution data. In the cases for which the separation shock was visible in the shadowgraph pictures, the point at which the pressure rise was detected corresponded reasonably well with the intersection of the separation shock with the wall.

Data with laminar boundary layers are shown in Figs. 12 and 13. These data show a much more pronounced pressure rise far upstream of the injector than the corresponding turbulent data; for example, compare data of Figs. 11 and 12 which were obtained at Mach number 2.61 and which have similar scale heights. Downstream of the injector, the data are similar for both boundary-layer states. In general, the normalized pressure distributions with either a laminar or a turbulent boundary layer are surprisingly insensitive to Mach number.

In spite of the good general correlation of the pressure data in Figs. 10-13, some systematic variations with pressure ratio or scale can be noted. In the case of laminar separation (Figs. 12 and 13), it can be seen that the separation distance normalized by $h$ decreases as $h$ increases. Considering the assumptions of the analytic model, it is not surprising that it does not account for an effect of shock/boundary-layer interaction particularly well. However, in the case of the turbulent boundary-layer data upstream of the injector, the correlation is excellent, and thus it appears that the turbulent separation distance is a linear function of $h$.

In Figs. 10-13, it can be seen that the agreement in the data is very good immediately downstream of the injection orifice in the region of minimum pressure, but a systematic difference appears somewhat farther downstream in the range of $(x / h)$ between 3 and 5 . As $h$, or the injection pressure, increases, the pressure in this region rises more abruptly, until it actually overshoots the freestream static pressure ex-

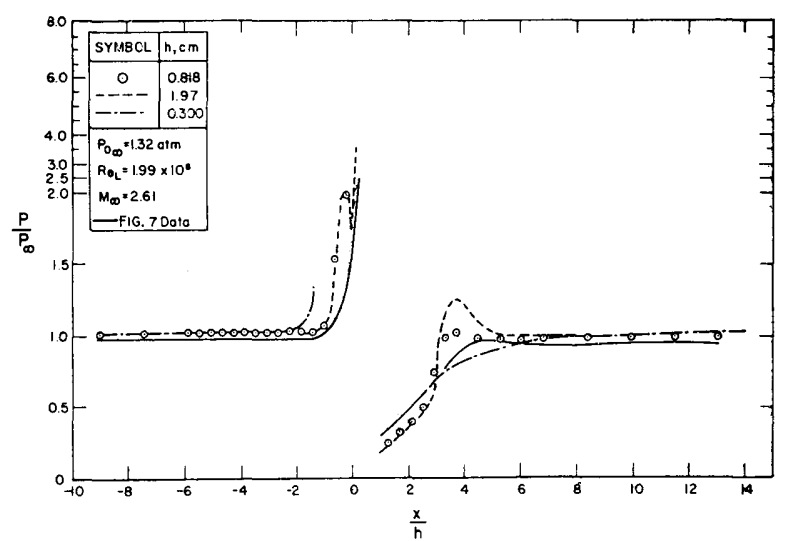

Fig. 11 Flat-plate static-pressure measurements in the plane $(y / h)=0$ at $M_{\infty}=2.61$ with nitrogen injection: turbulent boundary layer. 
cept at Mach number 4.54. At the lower Mach numbers, this overshoot becomes more pronounced as the injection pressure is increased. This overshoot occurs considerably downstream of any visible feature in the shadowgraph pictures to which it might be attributed.

This pressure overshoot is probably caused by the reattachment of the injectant jet to the wall. The increase in overshoot pressure with penetration height is explained by the fact that $h$ is increased by increasing the total pressure $P_{0 j}$. As $h$ increases, the pressure ratio through which the injectant expands, i.e., $P_{0_{i}} / P_{\infty}$, increases, and the velocity in the jet must increase. Hence, the turning shock must be stronger, and the pressures produced by the shock must be higher.

In each set of data at a constant Mach number, it was noted that the centerline pressure distribution corresponding to the lowest injection pressure seemed to be somewhat smeared out compared to the others. The pressure changes were more gradual, but extended over a larger region, again in the normalized coordinates. Although no detailed boundarylayer studies have yet been made to confirm this supposition, the schlieren and shadowgraph pictures seem to support the notion that this smearing out of the pressure distribution occurs when the scale of the obstruction is of the same order as a characteristic boundary-layer thickness. Since the scaling procedure is based upon a single scaling parameter for the flow field, it seems logical that this simplicity would be modified in a region where the scale factor was of the same order as another important characteristic dimension of the flow.

The correlation of the CIT data in Fig. 7 is not as good as that of the JPL data presented in Figs. 10-13 which appears to be excellent. At least some of the scatter in the CI'T results is due to the rather crude pressure instrumentation. The data for one of the higher pressure-ratio runs of the CIT data with a laminar boundary layer exhibit what appears to be the pressure plateau characteristic of laminar separation. This pressure plateau does not show up clearly in all of the CIT laminar boundary-layer data, as can be seen in Fig. 7 . It is not known whether this is entirely because of inaccuracies in measurement or whether the much more pronounced effects of laminar separation exhibited in Fig. 12 are primarily a result of actual differences in the shock/boundary-layer interaction between the two sets of experiments. In any event, the character of the boundary layer on the tunnel wall in the CIT tunnel would be expected to be different from that in the flat-plate experiments in the JPL tunnel.

Several papers have appeared in the literature which present pressure distributions on flat plates with secondary injection which are similar to the experiments described in this paper. ${ }^{2,6-8}$ Data from the paper by Cubbison, Anderson, and Ward $^{6}$ were considered to be the most directly compar-

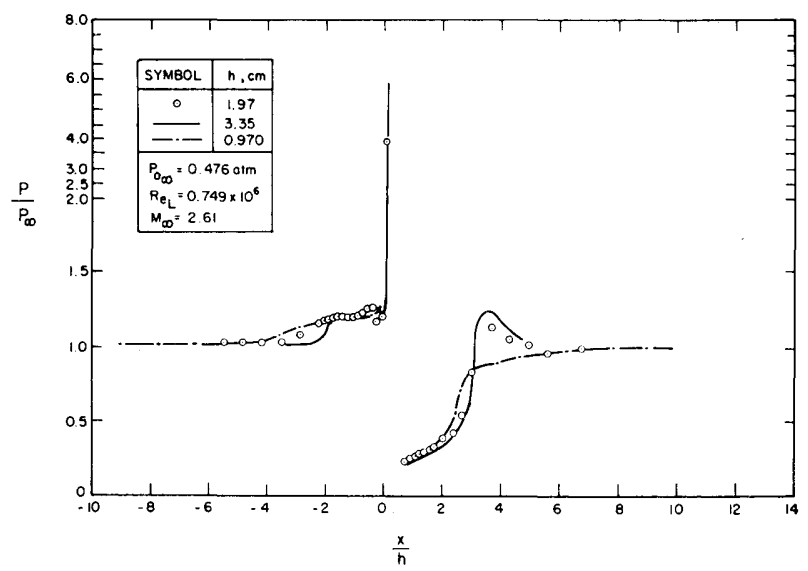

Fig. 12 Flat-plate static-pressure measurements in the plane $(y / h)=0$ at $M_{\infty}=2.61$ with nitrogen injection: laminar boundary layer.

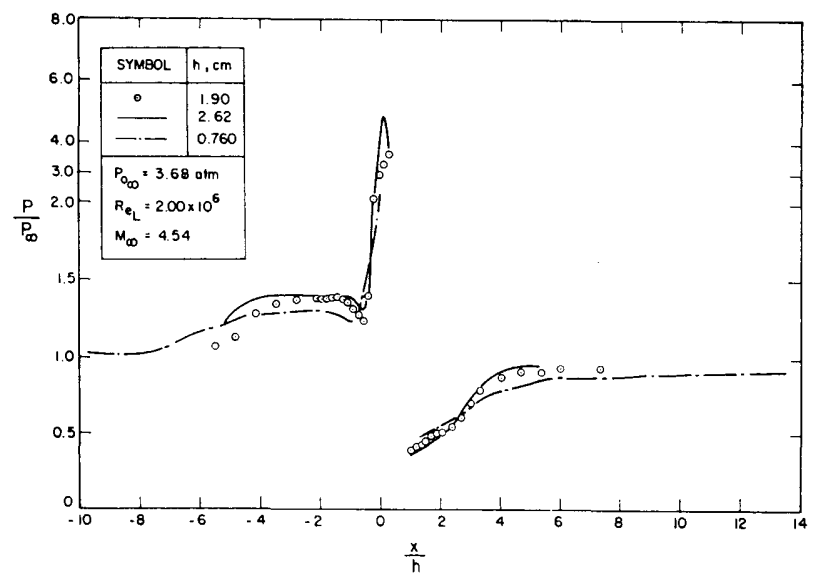

Fig. 13 Flat-plate static-pressure measurements in the plane $(y / h)=0$ at $M_{\infty}=4.54$ with nitrogen injection laminar boundary layer.

able to that which have been presented here. Pressure distributions obtained along the $x$ axis for $M_{\infty}=2.92$ and $M_{\infty}=$ 4.84 were normalized in the manner of Figs. 7 to 13 . The agreement was found to be quite good, except in the separated region upstream of the injector at freestream Mach number 4.84. For the two largest values of $h$, at $M_{\infty}=4.84$, the boundary layer was separated up to the leading edge of the plate, thus precluding any similarity in that region.

\section{Discussion and Conclusion}

Quantitatively, the results of the shock shape, concentration, and pressure measurements indicate that the scaling parameter $h$ is satisfactory for the range of variables which has been investigated, with the previously mentioned restriction that the penetration height be large with respect to the separated boundary-layer thickness. The correlation of the shock and concentration data was excellent. Some lack of similarity in the pressure data was observed, primarily in the laminar boundary-layer separation region and at the lower Mach numbers in the reattachment region downstream of the injector. It is also apparent that a simple oneparameter scale transformation cannot give a detailed correlation of the pressure data in these regions.

\section{Scaling Laws for Side Force on a Wall}

If the scaling law, as developed in Eq. (3), can be taken as being a good approximation, then it is easy to predict the variation of the side force generated on an infinite flat surface by the variation of the jet parameters. For many purposes, it is desirable to know the change in force produced on the wall by secondary injection.

The side force contribution from the pressure field resulting from secondary injection from an infinite flat plate can be expressed as

$$
\Delta F=\int_{x=-\infty}^{\infty} \int_{y=-\infty}^{\infty}\left(P-P_{\infty}\right) d x d y
$$

Dividing through by $\left(P_{\infty} h^{2}\right)$, we have

$$
\frac{\Delta F}{P_{\infty} h^{2}}=\int_{x / h=-\infty}^{\infty} \int_{y / h=-\infty}^{\infty} \frac{P-P_{\infty}}{P_{\infty}} \frac{d x}{h} \frac{d y}{h} \equiv \Phi
$$

The integral of Eq. (7) is evaluated in the normalized coordinates, and therefore will depend only upon the freestream Mach number and specific heat ratio. That is,

$$
\Phi=\Phi\left\{M_{\infty}, \gamma_{\infty}\right\}
$$

Using Eq. (5), we have

$$
\Delta F \propto m_{j}\left(R_{j} T_{0 j}\right)^{1 / 2} \Phi\left\{M_{\infty}, \gamma_{\infty}\right\}
$$


for $P_{\infty} / P_{0 i} \ll 1$. To this degree of approximation, $F_{i}$, the thrust of a sonic jet, can be written

$$
F_{j} \propto m_{j}\left(R_{j} T_{0 j}\right)^{1 / 2}
$$

The total normal force due to injection $F_{s}$ is the sum of the interaction force $\Delta F$ and the thrust of the sonic jet

$$
F_{\bullet}=F_{j}+\Delta F^{\prime} \propto m_{j}\left(R_{j} T_{0 j}\right)^{1 / 2}
$$

This result applies for a given set of primary flow condition.

It is desirable to put these results in a form suitable for the correlation of rocket motor test data. The usual form for presentation of rocket motor tests has been to give the ratio of side force to axial force as a function of the ratio of secondary to primary mass flow rates. That is,

$$
F_{\bullet} / F_{p}=f\left(m_{j} / m_{p}\right)
$$

If changes in axial thrust of the primary flow caused by nonoptimum expansion and by secondary injection are neglected, then

$$
F_{p} \propto m_{p}\left(R_{p} T_{0 p}\right)^{1 / 2}
$$

and therefore

$$
\left(\frac{F_{i}}{F_{p}}\right) \propto \frac{m_{i}}{m_{p}}\left[\frac{R_{i} T_{0 j}}{R_{p} T_{0 p}}\right]^{1 / 2}=\frac{m_{j}}{m_{p}}\left[\frac{T_{0 j} / \mathfrak{M}_{i}}{T_{0 p} / \mathfrak{M T}_{p}}\right]^{1 / 2}
$$

This result indicates that the thrust ratio depends directly on the mass flow rate and on the square root of the ratio of injectant total temperature to molecular weight. The ratio is independent of injector diameter.

The discussion given in the earlier sections of this paper indicates that there are a number of restrictions that must be placed on this scaling procedure. Clearly, the procedure is strictly applicable only if the wall on which the pressure disturbances exist includes the entire area of pressure disturbance. In practice, this means that the boundaries must be at least $10 h$ away from the injector.

In addition to this discussion of geometric limitation, it will also be useful to summarize the limitations on the scaling procedure itself. First, it is necessary that the penetration height be larger than the separated boundary-layer thickness.

Second, if the boundary layer is turbulent, scaling appears to be excellent except in the reattachment region. If scaling of a nozzle is carried out by using geometrically similar devices with equal total pressure ratios $\left(P_{0_{i}} / P_{0_{\infty}}\right)$, then the reattachment phenomena will also be similar and no scaling errors will be introduced. This scaling procedure is that which is most likely to be used in the design of a large rocket nozzle where the boundary layer is almost certainly turbulent.

Third, if the boundary layer is laminar, upstream separation phenomena are more important, and scaling with the penetration height may be less satisfactory. In this case, there is some indication that pressure changes compensate each other ${ }^{9}$; however, no information on such compensation can be deduced from the present work.

Some direct comparison can be made of the scaling law developed here with experimental rocket engine tests. Even though the flow field in a nozzle is not directly comparable to that treated here, it is felt that the general conclusions drawn from the present work are useful.

Rodriguez ${ }^{10}$ and many other experimenters have found that the side force is independent of the injector port area for fixed mass flow and depends linearly on the mass flow of injectant when the primary flow parameters are held fixed. This result agrees with that obtained from Eq. (10).

Some work has been carried out in which injectants with different total temperatures and different molecular weights were used. ${ }^{11-13}$ The correlation proposed by Lingen ${ }^{12}$ and later approximately verified by Chamay and Sederquist ${ }^{13}$ agrees exactly with Eq. (10).

Data from Refs. 11-13 for gas injection into a rocket nozzle have been correlated by the present technique. The correlations were quite good for the data of Refs. 12 and 13 and somewhat poorer for the data of Ref. 11. The different treatment proposed by Broadwell ${ }^{14,}{ }^{15}$ gives a somewhat better correlation of the data of Ref. 11, particularly at the higher injection rates. On the other hand, the molecular weight dependence that Broadwell predicts is different from that which was observed in the present series of wind-tunnel experiments. The difference in molecular weight dependence between the wind-tunnel and the rocket motor test data is believed to be a result of a difference in the relative importance of mixing of the secondary stream with the primary stream for the two situations. This comparison with rocket motor test data is presented in detail in Ref. 16, together with a discussion of assumptions that have been used by other workers in analyses of secondary injection phenomena, and a detailed derivation of the scaling law of Eq. (3) and its extension to a scaling law for the force on a flat plate.

\section{References}

1 Newton, J. F., Jr. and Dowdy, M. W., "Investigation of liquid and gaseous secondary injection phenomena on a flat plate with $M=2.01$ to $M=4.54$," Jet Propulsion Lab. TR $32-542$ (no date).

${ }^{2}$ Amick, J. L. and Hays, P. B., "Interaction effects of side jets issuing from flat plates and cylinders aligned with a supersonic stream," Wright Air Development Div. TR 60-329 (June 1960).

${ }^{3}$ Chernyi, G. G., Introduction to Hypersonic Flow, edited by R. F. Probstein (Academic Press, New York 1961), Chap. 3

${ }^{4}$ Lees, L., "Hypersonic flow," IAS Paper 554 (1955).

${ }^{5}$ Keffer, J. F. and Baines, W. D., "The round turbulent jet in a crosswind," J. Fluid Mech. 15, 481-496 (1963).

${ }^{6}$ Cubbison, R. W., Anderson, B. H., and Ward, J. J., "Surface pressure distributions with a sonic jet normal to adjacent flat surfaces at Mach 2.92 to 6.4," NASA TN D-580 (February 1961).

${ }^{7}$ Janos, J. J., "Loads induced on a flat-plate wing by an air jet exhausting perpendicularly through the plate and normal to a freestream flow of Mach number 2.0," NASA TN D-649 (March 1961).

${ }^{8}$ Lefko, W., "Loads induced on a flat plate at a Mach number of 4.5 with a sonic or supersonic jet exhausting normal to the surface," NASA TN D-1935 (July 1963).

${ }^{9}$ Ferrari, C., "Interference between a jet issuing laterally from a body and the enveloping supersonic stream," The Johns Hopkins Univ., Applied Physics Lab., Bumblebee Rept. 286 (April 1959).

${ }_{10}$ Rodriguez, C. J., "An experimental investigation of jetinduced thrust vector control methods," 17th Annual JANAFARPA-NASA Solid Propellant Meeting, Denver, Colo. (May 23-25, 1961).

${ }^{11}$ Walker, R. E., Stone, A. R., and Shandor, M., "Secondary gas injection in a conical rocket nozzle. I. Effect of orifice diameter and molecular weight of injectant," AIAA J. 1, 334338 (1963).

12 Lingen, A., "Jet-induced thrust-vector control applied to nozzles having large expansion ratios," United Aircraft Corp. Research Dept. Rept. R-0937-33 (March 1, 1957).

${ }^{13}$ Chamay, A. J. and Sederquist, R. A., "An experimental investigation of shock vector control with gaseous secondary injection," ARS Preprint 2216-61 (August 1961).

${ }^{14}$ Broadwell, J. E., "An analysis of the fluid mechanics of secondary injection for thrust vector control (revised)," Space Technology Labs. Rept. 6120-7744-MU-000 (March 15, 1962).

${ }^{15}$ Broadwell, J. E., "Correlation of rocket nozzle gas injection data," AIAA J. 1, 1911-1913 (1963).

${ }^{16}$ Zukoski, E. E. and Spaid, F. W., "Injection of gases into a supersonic flow," Guggenheim Jet Propulsion Center Rept., California Institute of Technology (October 1963). 